\title{
ETIMOLOGÍA DE LOS HUESOS DEL CUERPO HUMANO
}

Roy Riascos Castañeda, MD*, Juan Carlos Castro Navarro **

\section{Introducción}

La morfología moderna utiliza gran cantidad de herramientas nuevas para su aprendizaje dentro de las cuales se encuentran la multimedia, las disecciones interactivas y el internet, pero aún se usa la disección como base que permite la comprensión de la forma del cuerpo humano. La introducción de nuevas tecnologías muchas veces nos desvía de la morfología de las épocas de los anatomistas clásicos como Hipócrates y Galeno. Al revisar los nombres que se dieron a muchas estructuras a partir de raíces griegas y latinas, nos lleva a viajar en el tiempo para imaginar en qué estaban pensando los padres de la medicina al describir las diferentes partes del organismo. Este ejercicio es tan útil como las nuevas herramientas con que contamos para la manipulación de la información morfológica y nos permite utilizar la imaginación, gran aliada de la memoria.

Esperamos que esta revisión resulte tan entretenida para los lectores como para nosotros fue el descubrimiento del significado de los elementos óseos que conforman el cuerpo humano y que a su vez permita afianzar el conocimiento anatómico previo.

La mayor parte de las estructuras morfológicas del cuerpo humano reciben su nombre de su semejanza con artículos de la cotidianidad y tiene sus raíces del latín (Lat.) y el griego. (Gr.)

La comprensión de estas raíces permite una mejor aproximación para su aprendizaje. El cuerpo humano tiene 206 huesos constantes y el propósito de este artículo es hacer una revisión de las raíces etimológicas de los nombres de los huesos del cuerpo humano. Esperamos que esta información sea de utilidad para facilitar su aprendizaje a partir de la lógica del origen de sus nombres.

\footnotetext{
* Radiólogo, Hospital de San José, Instructor Asociado Sección de Morfología, Fundación Universitaria de Ciencias de la Salud.

** Estudiante, Facultad de Medicina, Fundación Universitaria de Ciencias de la Salud.
}

Hemos dividido por diferentes áreas el cuerpo. Primero tomaremos el cráneo y la cara y después describiremos en orden la etimología ósea del esqueleto axial, la pelvis superior, el miembro superior, la pelvis y el miembro inferior.

\section{Los huesos del cráneo}

Cráneo: (Gr.) kranion.

- Frontal: (Lat.) frente, se refiere a la parte anterior del cuerpo.

- Parietal: (Lat.) paries, pared.

- Occipital:(Lat.) occis, inicio y pitus, nuca

- Temporal: (Lat.) tempora, tiempo-sien. Lugar donde aparecen las primeras canas; otros autores lo atribuyen a su forma similar a la de un reloj de sol.

- Martillo: (Lat.) malleus, martillo.

- Yunque: (Lat.) incus, diente bicúspide.

- Estribo: (Lat.) stapes, estribo.

- Esfenoides: (Gr.) spheno y eidos, que asemeja. En forma de cuña.

- Pterigoides: (Gr.) pterigos, alas.

- Etmoides: (Gr.) ethmos, colador agujereado, criba y eidos, que asemeja.

- Papirácea: (Lat.) papyrus, papel.

- Cribosa: (Lat.) cribum, colador.

- Wormiano: relacionado o descrito por Ole Worm.

\section{Los huesos de la cara}

La cara comparte el esfenoides y el etmoides con el cráneo.

- Maxilar: (Lat.) maxilla, quijada.

- Mandíbula: (Lat.) mando, masticar.

- Cigomático: (Gr.) zygomatos, barra, pómulo. 


\section{Artículos de revisión}

\section{ETIMOLOGÍA DE LOS HUESOS DEL CUERPO HUMANO}

Roy Riascos Castañeda, MD *, Juan Carlos Castro Navarro **

\section{Introducción}

La morfología moderna utiliza gran cantidad de herramientas nuevas para su aprendizaje dentro de las cuales se encuentran la multimedia, las disecciones interactivas y el internet, pero aún se usa la disección como base que permite la comprensión de la forma del cuerpo humano. La introducción de nuevas tecnologías muchas veces nos desvía de la morfología de las épocas de los anatomistas clásicos como Hipócrates y Galeno. Al revisar los nombres que se dieron a muchas estructuras a partir de raíces griegas y latinas, nos lleva a viajar en el tiempo para imaginar en qué estaban pensando los padres de la medicina al describir las diferentes partes del organismo. Este ejercicio es tan útil como las nuevas herramientas con que contamos para la manipulación de la información morfológica y nos permite utilizar la imaginación, gran aliada de la memoria.

Esperamos que esta revisión resulte tan entretenida para los lectores como para nosotros fue el descubrimiento del significado de los elementos óseos que conforman el cuerpo humano y que a su vez permita afianzar el conocimiento anatómico previo.

La mayor parte de las estructuras morfológicas del cuerpo humano reciben su nombre de su semejanza con artículos de la cotidianidad y tiene sus raíces del latín (Lat.) y el griego. (Gr.)

La comprensión de estas raíces permite una mejor aproximación para su aprendizaje. El cuerpo humano tiene 206 huesos constantes y el propósito de este artículo es hacer una revisión de las raíces etimológicas de los nombres de los huesos del cuerpo humano. Esperamos que esta información sea de utilidad para facilitar su aprendizaje a partir de la lógica del origen de sus nombres.

\footnotetext{
Radiólogo, Hospital de San José, Instructor Asociado Sección de Morfología, Fundación Universitaria de Ciencias de la Salud.

** Estudiante, Facultad de Medicina, Fundación Universitaria de Ciencias de la Salud.
}

Hemos dividido por diferentes áreas el cuerpo. Primero tomaremos el cráneo y la cara y después describiremos en orden la etimología ósea del esqueleto axial, la pelvis superior, el miembro superior, la pelvis y el miembro inferior.

\section{Los huesos del cráneo}

Cráneo: (Gr.) kranion.

- Frontal: (Lat.) frente, se refiere a la parte anterior del cuerpo.

- Parietal: (Lat.) paries, pared.

- Occipital:(Lat.) occis, inicio y pitus, nuca

- Temporal: (Lat.) tempora, tiempo-sien. Lugar donde aparecen las primeras canas; otros autores lo atribuyen a su forma similar a la de un reloj de sol.

- Martillo: (Lat.) malleus, martillo.

- Yunque: (Lat.) incus, diente bicúspide.

- Estribo: (Lat.) stapes, estribo.

- Esfenoides: (Gr.) spheno y eidos, que asemeja. En forma de cuña.

- Pterigoides: (Gr.)pterigos, alas.

- Etmoides: (Gr.) ethmos, colador agujereado, criba y eidos, que asemeja.

- Papirácea:(Lat.) papyrus, papel.

- Cribosa: (Lat.) cribum, colador.

- Wormiano: relacionado o descrito por Ole Worm.

\section{Los huesos de la cara}

La cara comparte el esfenoides y el etmoides con el cráneo.

- Maxilar: (Lat.) maxilla, quijada.

- Mandíbula: (Lat.) mando, masticar.

- Cigomático: (Gr.) zygomatos, barra, pómulo. 
- Malar: (Lat.) mala, hueso de la mejilla.

- Ungüis: (Lat.) unguis, uña.

- Huesos propios nasales: (Lat.) nasus, nariz.

- Vómer: (Lat.) vomer, rastrillo metálico, reja de arado.

- Palatino: (Lat.) palatum, paladar, techo.

\section{Huesos del esqueleto axial}

- Columna vertebral: (Lat.) vertebratus, articulada. Vértebra: (Lat.) vertebrae, articulación y verto, voltear. Apófisis: (Gr.) apo, alejado de. Excrecencia ósea. Pedículo: (Lat.) base.

- Atlas: (Gr.) Atlas, titán de la mitología que sostiene al mundo sobre sus hombros, así como el atlas sostiene al cráneo sobre sus masas laterales.

- Axis: (Gr.) axon (Lat.) axis eje, superficie sobre la que se puede realizar un giro.

- Odontoides: (Gr.) odont, diente. En forma de diente.

- Sacro: (Lat.) sacrum, sagrado.

- Cóccix: (Gr.) kokkyx, cuchillo.

- Hioides: (Gr.) hyoeides, en forma de la letra ypsilon.

- Escápula: (Gr.) Espcapulae, espalda.

Acromion: (Gr )akromion, punta del hombro.

Coracoides: (Gr.) korakodes, en forma de pico de cuervo.

Glenoides (Gr.) Glenoieides, en forma de cavidad.

- Omoplato: (Gr.) omos, hombros.

- Clavícula: (Lat.) clavis, llave. Diminutivo de llave.

- Costilla: (Lat.) costa, adjetivo para costado.

- Esternón: modo latino, griego, sternon, pecho, torax.

- Coxal: (Lat.) coxa, cadera.

Ilion: (Lat.) ilium, ingle, flanco.

Isquion: (Gr.) ischion, cadera.

Pubis: (Lat.) pubes, vello de la parte inferior del vientre.

Acetábulo: (Lat.) acetabulum, vasija llana de vinagre.

\section{Miembro superior}

- Humero: (Lat.) humerus, hombro.

- Radio: (Lat.) radius, rayo, regla, vara.

- Cúbito: (Lat.) cubitus, codo.

- Ulna: (Lat.) ulna, codo, brazo.

Olécranon: (Gr.) olekranon, punta del codo.

Estiloides: (Gr.) stylos, pilar y eidos que asemeja.

- Carpo: (Lat.) karpos, en pedazos según Galeno.

Escafoides: (Gr.) skaphe, barco o lancha y eidos, que asemeja.

Navicular:(Lat.) navicularis, relacionado con un navío.

Semilunar: (Lat.) semi, media y luna, luna.

Piramidal: (Gr.) pyramis, en forma de estructura angulada (pirámide)

Pisiforme: (Lat.) pisum guisante. En forma de guisante.

Trapecio: (Gr.) tra cuatro, pous pies; trapezoin, mesa. Trapezoide: (Gr.) tra, cuatro, pous, pies; trapezoin, mesa y eidos, que asemeja.

Grande: (Lat.) capitalum, grande.

Ganchoso: (Lat.) hamalum, forma de anzuelo.

- Metacarpo:(Gr.) metha, más allá, mas allá del carpo.

- Falange: (Gr.) phalang, formación de batalla de soldados en línea unida.

\section{Miembro Inferior}

- Fémur: (Lat.) femur, muslo.

- Trocánter: (Gr.) trochanter, correr.

- Rótula:(Lat.) rotar, ruedecilla.

- Patela: (Lat.) plato, pequeño plato para sacrificios.

- Fabela:(Lat.) phabella, historieta, piececilla de teatro.

- Tibia:(Lat.) tibia, flauta.

- Peroné: (Gr.) broche.

- Fíbula: (Lat.) fibula, aquello que amarra, hebilla.

- Maléolo: (Gr)malleus, martillo. 
- Tarso: (Lat.) tarsus, superficie plana.

Calcáneo: (Lat.) calcar, espuela.

Astrágalo: (Gr.) astragalus, se decía del hombre que se sentaba en la segunda vértebra de un barco. Bola del tobillo.

Talo: (Lat.) talus, talón.
Cuboides: (Gr.) kybos, cubo y eidos, semejanza. En forma de cubo.

Cuña: (Lat.) cuneus, cuña.

- Metatarso: (Gr.) meta, más allá, mas allá del tarso.

- Sesamoideo: (Lat.) semilla del sésamo y eidos, que asemeja.

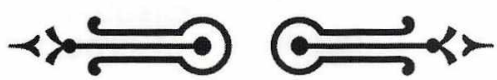

